\title{
People with lived experience at the centre of Canadian Stroke Best Practice Recommendations: A model for guideline developers
}

Patrice Lindsay ( $\triangle$ Patrice.Lindsay@heartandstroke.ca )

Heart and Stroke Foundation of Canada https://orcid.org/0000-0002-2584-8580

Natalie Gierman

Heart and Stroke Foundation of Canada

Jocelyn E. Harris

McMaster University

Gavin Arthur

Heart and Stroke Foundation of Canada

Moira E. Teed

Heart and Stroke Foundation of Canada

Anita Mountain

Dalhousie University

Gordon Gubitz

Dalhousie University

Eric E. Smith

University of Calgary

Dar Dowlatshahi

Ottawa Hospital Research Institute

Andrea de Jong

Heart and Stroke Foundation of Canada

Leigh C.P. Botly

Heart and Stroke Foundation of Canada

\section{Research}

Keywords: stroke, patient engagement, clinical practice guidelines; person-centred care; people with lived experience; stroke; rehabilitation; transitions of care; perceptions of care

Posted Date: January 10th, 2020

DOI: https://doi.org/10.21203/rs.2.20559/v1 
License: (c) (i) This work is licensed under a Creative Commons Attribution 4.0 International License. Read Full License

Version of Record: A version of this preprint was published at Journal of Patient Experience on September 10th, 2020. See the published version at https://doi.org/10.1177/2374373520956538. 


\section{Abstract}

\section{Background}

Actively engaging people with lived experience (PWLE) in stroke-related clinical practice guideline development has not previously been implemented and evaluated despite international efforts to incorporate patient and public engagement. The purpose of this pilot project was to evaluate the feasibility, perceived value and effectiveness of a new model, the Community Consultation and Review Panel, to actively engage PWLE in the writing and review of Canadian Stroke Best Practice Recommendations (CSBPR).

Methods

Members of the public with lived experience relevant to CSBPR module topics, including people with stroke, family members and caregivers, were recruited to participate in two CSBPR Community Consultation and Review Panels (CCRP). The CCRP ran in parallel to scientific writing groups updating two components of the CSBPR Rehabilitation, Recovery and Community Participation module Rehabilitation and Recovery following Stroke and Transitions and Community Participation following Stroke. With the aid of an inter-group liaison, both the scientific writing group and CCRP reviewed the updated evidence and CCRP participants added insights and context based on their personal experiences. We utilized the Patient and Public Engagement Evaluation Tool (PPEET) to obtain CCRP participant feedback.

Results

This model was found to be feasible, requiring 3-4 hours of staff time per week. CCRP participants rated "strongly agree" or "agree" to 14 PPEET questions indicating that they perceived the CCRP to be a positive experience and effective process, and their participation had an impact. Responses to the open-ended questions revealed that CCRP participants felt that their input and recommendations on the CCSPR were acknowledged and would benefit stroke care in Canada.

\section{Conclusions}

The overall success of this pilot project established the feasibility and perceived benefit of employing a participatory and collaborative model to actively engage PWLE in stroke-related clinical practice guideline development. The values, experiences and recommendations of PWLE were able to be effectively incorporated into CSBPR content to enable lived experience specific context and considerations to augment the existing scientifically rigorous writing and review process. This model is now the standard practice for all future CSBPR module development and updates and could be adapted for guideline development across other disciplines.

\section{Contributions To The Literature}


- Historically, clinical practice guidelines have been developed by health professionals and researchers. A fundamental aim of person-centred care is the active participation of people with lived experience (PWLE) in guideline writing and review, recognizing the value of the unique insights of PWLE with respect to their health condition and its treatment, as well as their ground-level experience navigating the healthcare system.

- Models of how to actively engage PWLE in guideline development are lacking and criticism has been directed at some approaches deemed too tokenistic. Moreover, participation of PWLE has not been formally evaluated. We developed and evaluated a new engagement model, the Community Consultation and Review Panel in the update of Canadian Stroke Best Practice Recommendations (CSBPR). This model established a reciprocal and collaborative knowledge sharing process between the expert scientific writing group and a PWLE community panel so that input from PWLE could be directly integrated into the updated guidelines.

- The results of this study address recognized gaps in the literature by providing a new and evaluated model to actively engage PWLE in guideline development that could be easily adopted by guideline developers across disciplines due to its straightforward methodology and low resource requirements. The values, experiences and recommendations of PWLE can effectively be incorporated into guideline development while maintaining the scientific rigor of the writing and review process.

\section{Background}

Over the past decade, there has been a concerted international effort to prioritize patient, family member and informal caregiver engagement at all stages of healthcare planning and delivery[1-5]. Adopting a person-centered care model leads to better patient experiences and perceived quality of care [6-11], and reduces healthcare utilization and costs [12]. A person-centred approach extends beyond the person experiencing the medical event or condition to include the perspectives and contributions of individuals, caregivers, families, and communities in all stages of the continuum of care[13]. A fundamental aim of person-centred care is the active participation of people with lived experience (PWLE) not only at the care planning stage, but also in planning research and in the development of clinical practice guidelines [14, 15]. This latter objective recognizes the value of the unique insights, experiential knowledge, and needs of PWLE with respect to their health condition and its treatment, personal goals and priorities, as well as their ground-level experience navigating the healthcare system.

Historically, clinical practice guidelines have been developed by health professionals and researchers to provide guidance for optimal patient care that is informed by an in-depth review of current evidence [16]. The most recent quality standards for guideline development put forth by international working groups strongly advocate for public and patient engagement in guideline writing and review $[17,18]$. However, determining the best method for this engagement in guideline development continues to be an avid avenue of study [14,19-21] as criticism has been directed at some approaches deemed too tokenistic $[19,22,23]$. Research has also begun to investigate the scope of its impact. For example, Young et al. (2015) found that twelve out of the thirteen Australian clinical practice guidelines published from 2006 to 
2014 included at least one patient-preference recommendation, such as statements directed at healthcare providers to encourage patients to actively participate in treatment plans and disease management [24]. There is evidence to suggest that engagement in guideline development is feasible and generally perceived as a positive experience for the participants involved [25-27]. However, models of how to engage PWLE in stroke-related guideline development are lacking and participation of PWLE has not been formally evaluated.

Funded and led by the Heart and Stroke Foundation of Canada (HSFC), the Canadian Stroke Best Practice Recommendations (CSBPR) are the primary evidence-based guidelines for stroke care across the continuum. The target audience for the CSBPR are healthcare professionals and health system leaders across all settings and phases of care. PWLE were engaged in the development and dissemination of previous editions of CSPBR using a variety of methods of engagement, including membership on the CSBPR advisory committee specific scientific writing groups, as well as by serving as external reviewers. Feedback on participant engagement, satisfaction and sense of overall impact were mixed using these approaches, and generally participants felt they were only peripherally engaged, often stating that they had little to contribute during discussions and that it was difficult to express their views freely. Therefore, a new approach was sought for the Sixth CSBPR edition to complement the HSFC's stronger strategic emphasis on partnerships with PWLE. Inputs from several advisory councils, including ones comprised equally of researchers, clinicians and PWLE, were operationalized to co-develop a new CSBPR engagement model. The Community Consultation and Review Panel (CCRP), a truly inclusive and integrated model of engagement for guideline development, was created recognizing that PWLE are able to provide unique insights into how their experiences and perspectives may inform new and updated recommendations, foster a person-centered approach to care, and incorporate validated and meaningful person-reported outcome and experience measures (PROMs, PREMs). The aim of this pilot project was to evaluate the feasibility, acceptability, perceived value and effectiveness of a new engagement model for people with lived experience in the update of the two components of the CSBPR Rehabilitation, Recovery and Community Participation module (Part One: Rehabilitation and Recovery following Stroke[28] and Part Two: Transitions and Community Participation following Stroke[29]).

\section{Methods}

\section{Design}

This pilot study of the feasibility, acceptability, perceived value, effectiveness of a CCRP was a nonrandomized mixed methods design, with purposeful recruitment of members. This study adhered to the Standards for Reporting Implementation Studies (StaRI) [30] (see Additional File 1). The CCRP model was built as a reciprocal and collaborative knowledge sharing process between the CSBPR scientific writing group and a community panel (CCRP) of PWLE (Fig. 1). The model was conceived by the CSBPR senior leaders in consultation with several PWLE who were participating on the CSBPR Advisory Committee and other HSFC advisory panels. A review of the literature was conducted to identify and assess other models of engagement of PWLE in guideline development and current evaluation evidence 
for those models. PWLE codeveloped the model and were consulted throughout the methodology development, the implementation of the process, and the evaluation. Data collection and evaluation included key informant interviews, informal discussions and online surveys.

\section{Community Consultation and Review Panel Participant Recruitment and Orientation}

A CCRP recruitment strategy was developed to ensure that participants collectively had lived experience relevant to the breadth of the module topic as a person with stroke or family member (e.g., attended inpatient rehabilitation; transitioned back to their home community, or cared for someone who met above criteria). Recruitment channels included the HSFC online peer support community of survivors and community of caregivers, provincial HSFC contacts, and recommendations from the relevant CSBPR scientific writing group members and existing advisory structures. Recruitment was purposeful when possible to attain CCRP members that were equally represented by sex, provinces, urban and rural areas, and included a combination of people who have had a stroke, as well as family members and caregivers of someone with stroke.

A list of possible participants was generated. A phone interview and short self-assessment screening questionnaire (see Additional File 2) were conducted to determine CCRP fit (i.e. to determine whether their goals for participation matched the goals of the project), eligibility and ability to actively participate in telephone and video-based meetings. Participants needed to be comfortable sharing their own experience in a group setting and be able to think broadly and beyond their own personal experiences to contribute constructive lessons learned and provide insights to the topics being discussed. Screening also confirmed members' motivation for participating, additional skills and expertise, such as group facilitation, clinical knowledge or editing that could be utilized during the CSBPR review process.

Eligible CCRP participants were provided with written offer letters and asked to sign conflict of interest and confidentiality forms, similar to the scientific writing group members. Participants attended an orientation session through an online webinar which introduced them to their role in the overall project, provided background information on the CSBPR writing and review process and its utility, included a more focused briefing on the specific CSBPR module topic (Rehabilitation and Recovery or Transitions and Community Participation), and interpretation of levels of evidence. The orientation session also provided an opportunity for CCRP members to build rapport, discuss their experiential stroke journey, and identify barriers and facilitators related to the module topics, that were then further explored through the subsequent meetings where relevant. Participants were expected to be able to keep all meeting materials confidential, and regularly attend meetings as required.

The first CCRP was created for the review of the CSBPR Part One: Rehabilitation and Recovery following Stroke[28]. The CCRP consisted of nine people who had a stroke, family members and caregivers, individually or as a couple, along with HSFC staff members and a liaison member of the scientific writing group. One person from each of the two CSBPR scientific writing groups volunteered to be the liaison 
between their respective CSBPR group and the CCRP to explain module recommendations, evidence and points of discussion by the scientific writing group as needed to the CCRP members and importantly, reciprocally share information and perspectives from the CCRP back to the scientific writing group.

Following completion of the final evaluation of the CCRP for Part One of the module, invitations were extended to participants to join the next CCRP for Part Two of the module, focused on Transitions and Community Participation following Stroke[29]. Seven of the original nine members accepted the invitation and one new person was recruited, for a total of eight members of the second CCRP. Reasons for not joining the second panel included a change in spouse availability to support participation $(n=1)$ and in personal circumstances $(n=1)$.

\section{Community Consultation and Review Panel Implementation Process}

Each CCRP ran in parallel to their respective scientific writing group. A member of the scientific writing group and a HSFC staff member served as the liaison between the CCRP and scientific writing group, sharing information and input back and forth. The CCRP pilot phase meetings were co-chaired by a HSFC staff member and a CCRP panel member. Each CCRP member participated in one-hour video conference calls. The first meeting coincided with the Research Phase of the CSBPR module update and provided an opportunity for CCPR members to be oriented to the review process. In the review phase meetings, the scientific writing groups reviewed the updated evidence and discussed revisions to recommendations. The updated information was then reviewed by the CCRP the following week where they added inputs based on their personal experiences. The format of the meetings included an overview of the section by the scientific writing group liaison, and then CCRP members asked questions of clarification, voiced their own experiences and proposed content or edits for areas they felt should be addressed or included to enhance care, facilitate recovery, and strategies to support the individual and their family for each topic as appropriate (e.g. access to rehabilitation, use of evidence-based interventions, or patient and family education). All meetings began and ended with a "check in" in order to ensure participants' thoughts and perceptions were captured and as an opportunity for further adjusting and refining the methodology.

Detailed notes were recorded by HSFC staff members, and the scientific writing group liaison reported on the feedback to the scientific writing group at their next meeting. Throughout, CCRP input was continuously integrated directly into the recommendations, the rationale, system implications and personreported outcome measures sections as appropriate. Initially the scientific writing group met every two weeks and the CCPR every three weeks, so as not to over burden the CCPR members; however, in order to integrate feedback in a timely and seamless way, meeting frequency for the CCRP was increased to every two weeks, on opposite weeks to the scientific writing group. Meetings of the scientific writing group and the CCPR continued until all sections of the module was discussed. Once the recommendation review phase was completed, CCRP members were engaged in ongoing activities for knowledge translation and dissemination. These included the co-development of resources and education tools (e.g. infographics and webinars) for health professionals, PWLE, their family members and caregivers. 


\section{Evaluation of Community Consultation and Review Panel Model}

Structured and informal evaluations took place at the midway point and at the completion of the first CCRP (Rehabilitation and Recovery following Stroke). Since most members of the first CCRP carried over to the second CCRP (Rehabilitation and Recovery following Stroke), a final evaluation only was conducted for the second CCRP. The data collected from the two panels were aggregated. We adapted the Patient and Public Engagement Evaluation Tool (PPEET), a validated public and patient evaluation tool [31] to evaluate our CCRP model. Briefly, the PPEET utilizes a questionnaire-based format to assess four key principles of high-quality public and patient engagement: 1. integrity of design and process, 2. influence and impact, 3. participatory culture, and 4. collaboration and common purpose.

At each evaluation timepoint, an anonymous online survey using the PPEET questionnaire was sent to all CCRP members to obtain feedback on the CCRP process. The survey included a questionnaire containing 14 closed-ended questions using a Likert scale and 4 open-ended questions. Survey questions assessed participants' level of satisfaction, perceived impact and effectiveness of the CCRP process, and included an opportunity for participants to provide written comments and suggest improvements to the model. At the completion of Part One and Two of the CSBPR module a final survey was also sent to each scientific writing group liaison to obtain feedback on the impact of the CCRP on the CSBPR writing and review process, and their personal experiences as a liaison. HSFC staff also provided feedback throughout the process. Subjective feedback from all participants was collected and documented throughout the pilot study.

\section{Results}

Participants: The first CCRP (Rehabilitation and Recovery following Stroke) was composed of 5 females (mean age: 41.8 years) and 4 males (mean age: 57.3 years) for a total of 7 patients and 2 caregivers from 7 Canadian provinces including Quebec. A total of 6 teleconferences were held with the group. The second CCRP (Transitions and Community Participation following Stroke) was composed of 5 females (mean age: 41.8 years) and 3 males (mean age: 52.5 years) for a total of 6 patients and 2 caregivers from 7 provinces. A total of 3 teleconferences were held with this group due to a smaller amount of content.

HSFC staff reported spending an average of three to four hours per week throughout the pilot study for participant recruitment and management, agenda and content preparation and meeting facilitation. Staff also spent time on notetaking, feedback gathering and synthesis of materials. The scientific writing group advisor reported spending on average one hour per week in preparation and participation on CCRP phone calls.

Evaluation of CCRP model: At the midway point of the first CCRP, seven of nine participants (78\%) completed the online survey. They all strongly agreed or agreed on most elements of the evaluation tool. 
One person rated disagree on having enough information to contribute to the discussion, attributed to the relevance to their own personal experience (e.g., they did not receive certain rehabilitation therapies and therefore could not comment on them). The only areas of concern were related to the technical quality of the teleconferences including sound and video access.

Six of the seven individual participants who were on both the first and second panels responded to the final evaluation of the CCRP model (75\% response rate); one individual member and the husband-wife pair were unable to complete the evaluation $(n=3)$. Figure 2 summarizes the responses of CCRP members to the 14 Likert scale questions from the final evaluation of the CCRP model. All participants who completed the evaluation agreed or strongly agreed that they were satisfied, felt their participation would make a difference and that the panel achieved its objective. The majority of respondents $(n=5$, $83 \%)$ strongly agreed that their views were heard, and all of the respondents strongly agreed $(n=3,50 \%)$ or agreed $(n=3,50 \%)$ that they were able to express their views freely.

Table 1 provides representative responses provided by CCRP members from both panels to the four openended questions from the final evaluation timepoint. Subjective feedback was positive, with CCRP members highlighting the value of meeting other PWLE, being able to share their stories in a safe environment, and having their experiences benefit those with stroke. Expressed concerns were mostly related to technical quality of the conference calls and the webinar program on their own computer systems, and one member where English was their second language. The inputs from the CCRP were integrated throughout Parts One and Two of the CSBPR module, particularly in the Rationale and System Implications sections $[28,29]$.

\section{Table 1. CCRP Model Evaluation: Representative Responses to open-ended questions.}




\section{Question}

1. How do you think the results of your participation will be used?

\section{Representative responses from CCRP Members}

"I believe comments made and noted during meetings and the comments received through individual feedback forms will be considered in updating the best practices documents. I realize not all comments will necessarily be used."

"I believe the inclusion of myself and my peers will reflect recovery from the stroke survivors' point of view. It's a great move forward to have diverse opinions from stakeholders in order to know if CSBP recommendations are having an effect. There will be consideration of the stroke survivor's needs."

"To inform/guide all that have experienced stroke with real life examples"

\section{What is the best thing about the Consultation and Review Panel?}

"A chance to share our story, our course for the good of others."

"I appreciate the work that has gone in to developing, maintaining and updating these documents as they are widely used across the country when developing stroke care strategies. It has been great talking with other survivors and care givers to get a sense of the variety of care that is experienced so perhaps to strengthen the recommendations and improve care across the country."

"Being able to voice concerns of stroke survivors. Feeling acknowledged for experience."

"Online consultation could somehow be made more user friendly for some of us who do not have very good computer skills."

\section{Please identify at least one improvement we could make for future review panel processes.}

"It would be nice to be able to have the sound of the meeting on the computer, not over a phone call."

"Difficulties of language for people like me with a poor English speaking."

4. Additional Comments

\begin{abstract}
"Glad to have participated and glad to see H\&S reach out to us."
\end{abstract}
"Enjoyed my time on these calls."

"Thank you for this opportunity."

The scientific writing group liaisons and program staff reported that CCRP input was included in as many stages of the process of guideline revision as possible. They rated strongly agree to questions regarding their interest in further training on public and patient engagement; and agreed that as a result of the 
experience they would be more comfortable in leading public and patient engagement activities in the future, and that the process was a good use of program resources and enhanced decision making.

\section{Discussion}

Engaging PWLE in stroke-related clinical practice guideline development in a systematic, meaningful and measurable way has not previously been implemented and evaluated despite internationally-driven efforts to standardize the inclusion of these groups $[17,18]$. In this pilot project, we developed and tested a new model and successfully engaged PWLE in the review and update of a CSBPR module, including two parts that fell within the umbrella group of Rehabilitation, Recovery and Community Participation following Stroke. The CCRP model established a reciprocal and collaborative knowledge sharing process between the CSBPR expert scientific writing group and a PWLE community panel (CCRP) at all stages of the CSBPR process, including review, writing, and dissemination. The CCRP model provided members with opportunities to regularly review and offer feedback on draft recommendations from the expert writing group, provide context related to their values and preferences in stroke care to help inform what person-centred care meant in the context of their lived experiences, and co-develop lay language knowledge translation resources and education tools for PWLE and caregivers.

The goal of the CCRP model was to facilitate development of specific stroke best practice recommendations that are grounded in real-life experience and the unique needs of people with stroke while maintaining scientific rigour, and applicability to those directly impacted by the recommendations people who have had a stroke, their families and caregivers. Incorporating feedback from the CCRP helped to highlight areas within the guidelines that are considered to be of high importance and value by PWLE, family members and caregivers. Health professionals who use these guidelines can use this feedback to provide a person-and-family centred approach.

Prior to implementation of this engagement model, two or three PWLE would participate as members on the full CSBPR writing groups along with representatives from a range of professional groups involved in stroke care. In addition, two to three PWLE were asked to review the final module as part of the external review process. The PWLE members of the writing groups were often disengaged, and in one-on-one feedback discussions with them they stated that they felt they had little to contribute during the discussions about the available evidence, it was difficult to speak up during conference calls, and they sometimes felt dismissed by other writing group members when they did comment. The CCRP model was designed to mitigate these issues and provide opportunities for CCRP members to participate more fully in an encouraging and accepting environment, at a pace and level of discussion appropriate to their understanding, and in a setting that was less intimidating. The liaison member of the scientific writing group was a key role, which was filled by an occupational therapist during the first stage of the pilot. The HSFC staff leads were critical as well to implement the processes and to ensure that the voices of PWLE were heard by the scientific writing group and that their feedback was incorporated throughout the final module development. The staff members were then responsible, along with the scientific writing group liaison, to actually integrate CCRP inputs into all appropriate sections of the guidelines during the writing phase. 
Feedback using the PPEET tool indicated that we met our goals for the CCRP. Respondents to the evaluation perceived it to be a positive experience and effective process and felt that their participation had an impact (Fig. 2). Importantly, all respondents strongly agreed or agreed that that they were able to express their views freely and that their views were heard. This is a substantial improvement when compared to the feedback obtained from PWLE using previous engagement techniques. Responses to the open-ended questions indicated that CCRP participants felt that their input and recommendations on the CCSPR were acknowledged and would benefit stroke care in Canada (Table 1). For example, respondents commented that the CCRP process was an inclusive activity that incorporated the points of view of people with stroke, their needs, and real-life experiences. Respondents felt that their feelings and experiences were acknowledged, and they enjoyed the opportunity to connect and hear the experiences of other PWLE across the country to develop stroke recovery strategies and recommendations. It was meaningful for CCRP participants to know that their efforts would help improve the recovery process of others who have experienced a stroke. Respondents made several recommendations for improvement to the CCRP process, including providing additional resources for participants who are not technologically savvy and for additional language resources for those whose primary language is not English. Some respondents commented that they were less sure of the impact their engagement might have. This is likely a result of the evaluation occurring before the module was finalized and published. This provides an opportunity for the research team to re-engage CCPR members in further dialogue at the time of publication; calling upon them to participate in webinars and other knowledge translation activities.

This study was a pilot project that had some limitations. The two panels included a relatively small number of volunteer CCRP participants, since many stayed for both rounds (Parts One and Two of CSBPR module). Thus, the generalizability of the positive experiences and feedback reported by CCRP participants to the larger community of PWLE is uncertain. It is also important to note that none of the CCRP members were residents of the northern territories, although a few were from more rural regions within their Provinces. Future implementation of this model will strive to include representation from rural and remote regions in Canada to best capture any gaps or issues related to stroke care across Provinces and Territories. The CSBPR advisory committee has now approved the inclusion of CCRPs for all new and updated modules as part of the Seventh Edition of the CSBPR. Through these additional panels, we will continue to refine implementation and evaluation of this model of engaging PWLE in CSBPR writing and review, and the development of resources that explain the stroke best practices to PWLE so they can more fully engage in their recovery as partners and not just receivers of care.

\section{Conclusions}

The overall success of this pilot project established the feasibility and perceived benefit of employing a participatory and collaborative model, the CCRP, to actively engage PWLE in clinical practice guideline development, including all stages from writing to review and dissemination/knowledge translation. The results indicate that the CCRP increases PWLE engagement and provides guideline users with valuable evidence-based recommendations supported by personal experiences, insights and feedback. We have shown that the values, experiences and recommendations of PWLE can effectively be incorporated into 
CSBPR development while maintaining the scientific rigor of the writing and review process, and provide a richer set of guidance for practitioners and system planners. This model could be adapted for guideline development across disciplines. Our research group aims to continue implementation of the CCRP model in the update of future CSBPR to obtain further evaluative data, expand the scope of its implementation and enhance its impact.

\section{Abbreviations}

CCRP

Community Consultation and Review Panel

CSBPR

Canadian Stroke Best Practice Recommendations

HSFC

Heart and Stroke Foundation of Canada

PPEET

Patient and Public Engagement Evaluation Tool

PWLE

person with lived experience

\section{Declarations}

\section{Ethics approval and consent to participate}

Ethics approval was not required for this pilot study. All CCRP participants provided consent to participate and completed a conflict of interest declaration prior to participation. All members of the CSBPR scientific writing group completed declarations of conflict of interest at the outset of the process.

\section{Consent for publication}

Not applicable.

\section{Availability of data and materials}

The datasets used and/or analyzed during the current study are available from the corresponding author on reasonable request.

\section{Competing interests}

No authors had competing interests directly related to this research. The following participants had unrelated conflicts to declare: Dar Dowlatshahi received honoraria from Bayer, BMS, Apopharma; Gord Gubitz is a member of the advisory boards for Bayer, Boehringer Ingelheim and Pfizer; the remaining contributors had no unrelated conflicts to declare. MPL, NG, GA, MT, ADJ and LB are current employees of the Heart and Stroke Foundation of Canada. 


\section{Funding}

The development of the CSBPR, including the CCRP model was funded in its entirety by the Heart and Stroke Foundation of Canada. No funds for the development of this work were received from commercial interests, including pharmaceutical and medical device companies. All members of the scientific writing groups and CCRP were volunteers and did not receive any remuneration for participation in guideline development, updates and reviews.

\section{Authors' contributions}

PL was the project lead, conceived of the CCRP model and initial design and was involved in all aspects of implementation, evaluation and writing of this manuscript. NG participated in the initial CCRP model design, and led the evaluation process. GA and MT contributed to CCRP model development, and MT conducted recruitment of the CCRP participants and co-facilitated the pilot CCRP teleconferences. JH acted as the liaison between the CCRP and the Rehabilitation and Recovery scientific writing group, and contributed to refining the design of the CCRP model. EES, AM and GG are CSBPR advisory committee leaders and were involved in initial design of the CCRP module and review of this manuscript. ADJ provided review and edits to the manuscript. LB was a major contributor to the writing of the manuscript. All authors provided review, input and final approval of this manuscript.

\section{Acknowledgements}

The Heart and Stroke Foundation of Canada (HSFC) is especially grateful to the members of the Community Consultation and Review Panel who reviewed all sections of this module, shared their personal experiences and insights on what did or would have made their journey optimal. The members of both the Rehabilitation and Recovery following Stroke and Transitions and Community Participation following Stroke CCRPs included: Steven Archer, Rob Claydon (co-facilitated Transitions and Community Participation CCRP), Debbie Chow, Daniel Franco, Amanda Horner, Bruce Hughes, Edith Lambert, Cathy Livingstone, David Livingstone and Michelle McGroty. The HSFC gratefully acknowledges the members of the CSBPR scientific writing groups for Rehabilitation and Recovery following Stroke and the Transitions and Community Participation following Stroke all of whom have volunteered their time and expertise to the update of these recommendations and participate in the development of the CCRP model and integration of the CCRP feedback into the final module content. We thank the Canadian Stroke Best Practices and Quality Advisory Committee members for their support and input throughout the development and pilot testing of the CCRP model.

\section{References}

1. Brett J, Staniszewska S, Mockford C, Herron-Marx S, Hughes J, Tysall C, et al. A systematic review of the impact of patient and public involvement on service users, researchers and communities. The Patient. 2014;7:387-95. 
2. Ocloo J, Matthews R. From tokenism to empowerment: progressing patient and public involvement in healthcare improvement. BMJ Qual Saf. 2016;25:626-32.

3. Brett J, Staniszewska S, Mockford C, Herron-Marx S, Hughes J, Tysall C, et al. Mapping the impact of patient and public involvement on health and social care research: a systematic review. Health Expect Int J Public Particip Health Care Health Policy. 2014;17:637-50.

4. Baker GR, Fancott $\mathrm{C}$, Judd M, O'Connor P. Expanding patient engagement in quality improvement and health system redesign: Three Canadian case studies. Healthc Manage Forum. 2016;29:176-82.

5. Mockford C, Staniszewska S, Griffiths F, Herron-Marx S. The impact of patient and public involvement on UK NHS health care: a systematic review. Int J Qual Health Care J Int Soc Qual Health Care. 2012;24:28-38.

6. McMillan SS, Kendall E, Sav A, King MA, Whitty JA, Kelly F, et al. Patient-centered approaches to health care: a systematic review of randomized controlled trials. Med Care Res Rev MCRR. 2013;70:567-96.

7. Laine C, Davidoff F. Patient-Centered Medicine: A Professional Evolution. JAMA. 1996;275:152-6.

8. Kitson A, Marshall A, Bassett K, Zeitz K. What are the core elements of patient-centred care? A narrative review and synthesis of the literature from health policy, medicine and nursing. J Adv Nurs. 2013;69:4-15.

9. Ulin K, Malm D, Nygårdh A. What Is Known About the Benefits of Patient-Centered Care in Patients with Heart Failure. Curr Heart Fail Rep. 2015;12:350-9.

10. Stewart M, Brown JB, Donner A, McWhinney IR, Oates J, Weston WW, et al. The impact of patientcentered care on outcomes. J Fam Pract. 2000;49:796-804.

11. Ekman I, Swedberg K, Taft C, Lindseth A, Norberg A, Brink E, et al. Person-Centered Care - Ready for Prime Time. Eur J Cardiovasc Nurs. 2011;10:248-51.

12. Bertakis KD, Azari R. Patient-centered care is associated with decreased health care utilization. J Am Board Fam Med JABFM. 2011;24:229-39.

13. World Health Organization. WHO | Health Systems Strengthening Glossary [Internet]. WHO. [cited 2019 Sep 4]. Available from: https://www.who.int/healthsystems/hss_glossary/en/index8.html\#2

14. Armstrong MJ, Rueda J-D, Gronseth GS, Mullins CD. Framework for enhancing clinical practice guidelines through continuous patient engagement. Health Expect Int J Public Particip Health Care Health Policy. 2017;20:3-10.

15. Loudon K, Santesso N, Callaghan M, Thornton J, Harbour J, Graham K, et al. Patient and public attitudes to and awareness of clinical practice guidelines: a systematic review with thematic and narrative syntheses. BMC Health Serv Res. 2014;14:321.

16. Institute of Medicine (US) Committee to Advise the Public Health Service on Clinical Practice Guidelines. Clinical Practice Guidelines: Directions for a New Program [Internet]. Field MJ, Lohr KN, editors. Washington (DC): National Academies Press (US); 1990 [cited 2019 Mar 1]. Available from: http://www.ncbi.nlm.nih.gov/books/NBK235751/ 
17. Brouwers MC, Kho ME, Browman GP, Burgers JS, Cluzeau F, Feder G, et al. AGREE II: advancing guideline development, reporting and evaluation in health care. Can Med Assoc J. 2010;182:E83942.

18. About the G-I-N PUBLIC Toolkit: Patient and Public Involvement in Guidelines - Guidelines International Network [Internet]. [cited 2019 Feb 21]. Available from: https://www.g-i-n.net/workinggroups/gin-public/toolkit

19. van Wersch A, Eccles M. Involvement of consumers in the development of evidence based clinical guidelines: practical experiences from the North of England evidence based guideline development programme. Qual Health Care QHC. 2001;10:10-6.

20. Armstrong MJ, Mullins CD, Gronseth GS, Gagliardi AR. Recommendations for patient engagement in guideline development panels: A qualitative focus group study of guideline-naïve patients. PloS One. 2017;12:e0174329.

21. Díaz Del Campo P, Gracia J, Blasco JA, Andradas E. A strategy for patient involvement in clinical practice guidelines: methodological approaches. BMJ Qual Saf. 2011;20:779-84.

22. Hahn DL, Hoffmann AE, Felzien M, LeMaster JW, Xu J, Fagnan LJ. Tokenism in patient engagement. Fam Pract. 2017;34:290-5.

23. Tong A, Lopez-Vargas $P$, Howell M, Phoon R, Johnson D, Campbell D, et al. Consumer involvement in topic and outcome selection in the development of clinical practice guidelines. Health Expect Int $\mathrm{J}$ Public Particip Health Care Health Policy. 2012;15:410-23.

24. Young CE, Boyle FM, Brooker KS, Mutch AJ. Incorporating patient preferences in the management of multiple long-term conditions: is this a role for clinical practice guidelines? J Comorbidity. 2015;5:122-31.

25. Harding E, Brown D, Hayward M, Pettinari CJ. Service user perceptions of involvement in developing NICE mental health guidelines: A grounded theory study. J Ment Health Abingdon Engl. 2010;19:249-57.

26. Simpson EL, House AO. Involving users in the delivery and evaluation of mental health services: systematic review. BMJ. 2002;325:1265.

27. Serrano-Aguilar P, Trujillo-Martin M del M, Pérez de la Rosa A, Cuellar-Pompa L, Saavedra-Medina $H$, Linertova R, et al. Patient participation in a Clinical Guideline Development for Systemic Lupus Erythematosus. Patient Educ Couns. 2015;98:1156-63.

28. Canadian Stroke Best Practice Recommendations: Rehabilitation, Recovery and Community Participation. Part One: Rehabilitation and Recovery following Stroke, 6th edition update 2019. Submitted to International Journal of Stroke (November 11, 2019).

29. Canadian Stroke Best Practice Recommendations: Rehabilitation, Recovery and Community Participation. Part Two: Transitions and Community Participation following Stroke, 6th edition update 2019. Submitted to International Journal of Stroke (November 11, 2019).

30. Pinnock H, Barwick M, Carpenter CR, Eldridge S, Grandes G, Griffiths CJ, et al. Standards for Reporting Implementation Studies (StaRI) Statement. BMJ. 2017;356:i6795. 
31. Abelson J, Li K, Wilson G, Shields K, Schneider C, Boesveld S. Supporting quality public and patient engagement in health system organizations: development and usability testing of the Public and Patient Engagement Evaluation Tool. Health Expect Int J Public Particip Health Care Health Policy. 2016;19:817-27.

\section{Figures}
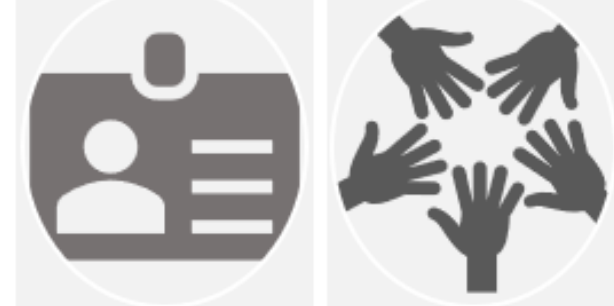

\section{Onboarding}

- Phone interviews

- Psychometric selfassessment screening

- Offer letters, confidentiality and conflict of interest completion

- Introductions

- Background and information on CSBPR writing and review

- Experiential journey discussion

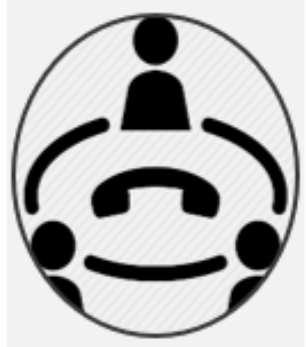

Regular Meetings

- Facilitators \& barriers discussion

- Re-cap and check-ins (PWLE Co-Facilitator)

- Review of module topics and recommendation $s$, receive inputs and suggestions

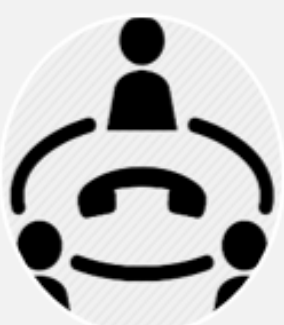

Final Meeting

- Midpoint evaluation

- Re-cap and check-ins (PWLE Co-Facilitator)

- Review progress and accomplishments

- Sumarize inputs

- Final check-in (PWLE CoFacilitator) and
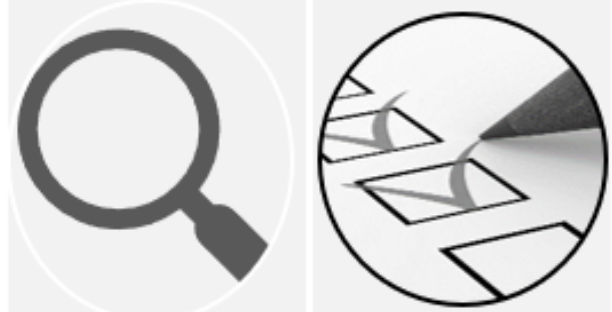
subjective

\section{Final}

content review

- Input on knowledge translation resources

- Sharing final draft module

\section{Evaluation and Feedback}

- Final evaluation using PREET

\section{Figure 2}

Community Consultation and Review Panel Model. 
1. The purpose of the panel was clearly explained

2. The supports I needed to participate were available

3. I had enough information to contribute to the topic being discussed

4. I was able to express my views freely

5. I feel that my views were heard

6. A wide range of views on the topic were expressed

7. I feel that the input provided through this activity will be considered by Heart and Stroke

8. The panel is achieving its stated objectives

9. I understand how the input from this panel will be used

10. I think this activity will make a difference

11. As a result of my participation in this panel, I am better informed about the CSPBR and the writing process

12. As a result of my participation in this activity I have a greater trust in Heart and Stroke

13. Overall I am satisfied with this activity

14. This activity was a good used of my time

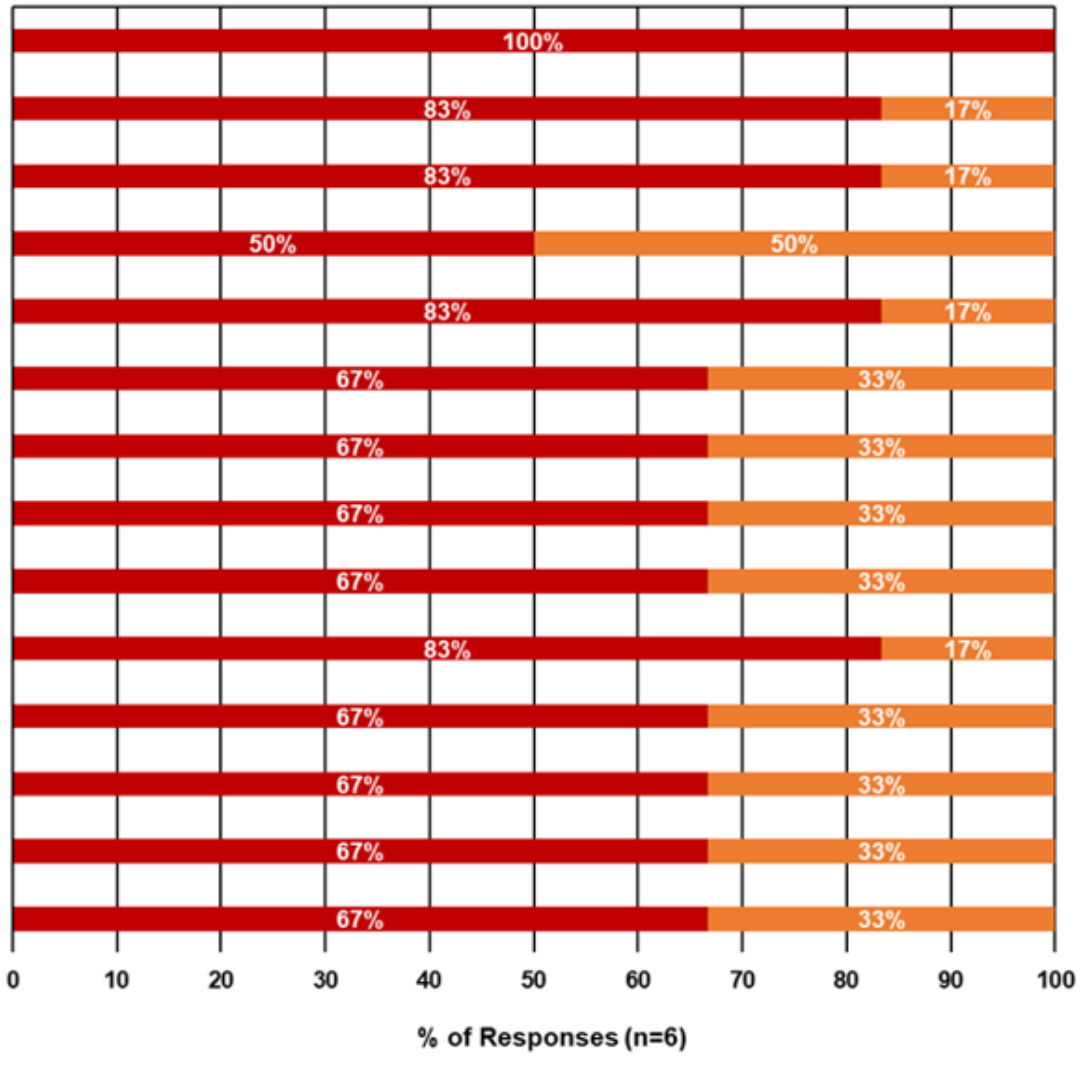

-Strongly Agree $=$ Agree $=$ Neither Agree nor Disagree $=$ Disagree $\equiv$ Strongly Disagree

\section{Figure 3}

Evaluation of the CCRP model. Responses to the Likert scale questionnaire administered to Community Consultation and Review Panel (CCRP) members during the final evaluation of the model.

\section{Supplementary Files}

This is a list of supplementary files associated with this preprint. Click to download.

- AdditionalFile2.doc

- AdditionalFile1.docx

- AdditionalFile2.doc

- AdditionalFile1.docx 